\title{
CONCEPT AND EVALUATION OF AN URBAN PLATFORM FOR INTERACTIVE VISUAL ANALYTICS
}

\author{
Hanadi Ebrahim*, Thunyathep Santhanavanich, Patrick Wuerstle, Volker Coors \\ University of Applied Sciences Stuttgart \\ Schellingstraße 24 \\ 70174 Stuttgart, Germany \\ hanadi92ebrahim@gmail.com,(thunyathep.santhanavanich, patrick.wuerstle, volker.coors)@hft-stuttgart.de
}

\begin{abstract}
KEY WORDS: Urban Platform, 2D/3D Data Integration, Analytic Visualization, Interactive Visualization, 3D City Models, En-
\end{abstract} vironment, Traffic, Pollutants Emissions.

\begin{abstract}
:
Urban platforms are becoming a vital role player in city resources management for achieving the right balance between social and economic services and their impact on the environment. More and more cities are starting to benefit from an urban platform to state the city conditions and re-coin the shape of life depending on data gathered from different city systems. However, urban platforms need further support of data analytics in respect of reaching a smart city platform helping city planners with better decision making. Besides, the majority of operational urban platforms consider 2D data only, missing out on the possible information that could be obtained from $3 \mathrm{D}$ city models. This work proposes a concept for an urban platform that supports data manipulation and visualization plus interactive analytic functionalities. As a prototype, an urban platform is implemented for integrating and processing $3 \mathrm{D}$ city models and 2D traffic data to derive air pollutants emission rates with analytic visualization, leading urban planning to concentrate on the most affected areas. In addition, interactive features are implemented, including filtering, querying, and classifying data to support the analytic visualization in the developed urban platform.
\end{abstract}

\section{INTRODUCTION}

The urban population is forecast to reach 6.419 billion by 2050 , with an increase of $66 \%$ (URBANET, 2016). This exponential urbanization raises challenges to provide services for city inhabitants with optimizing resources management and energy consumption as well as reducing the potential impact on the environment. In order to improve urban planning, cities are transforming into smart cities by implementing their own urban platforms. Thereupon, many studies have been carried out to set up standards and approaches for smart city implementation that guarantees the basic requirements of the urban platforms as openness, efficiency, quality of life, and interoperability (Hernandez et al., 2020). The substantial element of the urban platform concept is interoperability due to the integration of various systems with heterogeneous data sources. Data interoperability considers the vertical data flows between service layers and horizontal data flows between different applications (Brutti et al., 2019).

Although cities are steadily more converting into smart cities through digitization and urban platform establishment, the supported functionalities by the operational urban platforms are still narrow to sharing and visualizing data. As stated in a study conducted by Erasmus Centre for Data Analytics (ECDA) about urban data platform developments in 80 European cities, only $12 \%$ of urban platforms use $3 \mathrm{D}$ visualization for the twin city, and currently, urban platforms support a limited degree of analytics from sharing raw data without extracting new information from it. Furthermore, the study shines a light on urban platform contribution to the triple bottom line: people, planet, and profit. Urban platforms target mainly offering creative and efficient city operations, but with caution of external needs for people

\footnotetext{
* Corresponding author
}

and environmental sustainability (Tobias Brandt and Sheombar, 2020).

This research presents a concept of an urban platform to appraise two main aspects. Firstly, data integration among different dimensions 2D and 3D data sets is explored to define an effective way to implement it and determine which level of the system can manage this integration. Secondly, analyzing data is a considerable issue. It involves analyzing the used data sets (2D/3D) and obtaining added-value information about the study area. This information could be counted as valuable assets for establishing required strategies or recommendations to improve the urban area's state. To prove the concept, an urban platform is developed for the "Nordbahnhof" area in Stuttgart city, Germany, including data sets for 3D city models and 2D data about traffic. The paper is structured as follows. Section 2 includes an overview of the related work. Section 3 illustrates the overall workflow with the proposed concept and the implementation of the system parts is described in Section 4, followed by the evaluation of the proposed concept in Section 5. Finally, the conclusions and recommendations for future work are presented in Section 6.

\section{RELATED WORK}

\subsection{Urban Platform}

The urban platform concept has become a major concern to handle the massive amount of data generated from the different city sectors and from heterogeneous resources. Diverse approaches were proposed to enhance the efficiency of urban platforms. (Miu et al., 2018) introduces a system called $\mathrm{PlaniSphere} \mathrm{to} \mathrm{integrate} \mathrm{2D} \mathrm{spatial} \mathrm{data} \mathrm{into} 3 \mathrm{D}$ visualization using Service-Oriented Architecture (SOA) with Online 
Analytical Processing (OLAP) to provide analyzed spatial data formed as services. Other methodologies of structuring urban platforms are data federation and data encapsulation. Data federation is accomplished by using cloud hubs. CityHub discussed the different structures of hybrid cloud hubs and their ability to share Internet of Things (IoT) data, offering data access to multiple applications through the data hub (Lea and Blackstock, 2015). On the other hand, an encapsulation mechanism is applied to standardize information from different sources.Heterogeneous traffic sensors were unified into a connected network with a Multi-Agent System to build up a smart platform (Chamoso and De La Prieta, 2015).

An Urban platform is a crucial facet in promoting transparency and openness of urban data. Many implementations of this concept have been done in different domains such as traffic, environment, energy consumption, etc. In the Netherlands, an Urban Data Access Platform (UDAP) is developed to offer information about traffic from linked intelligent traffic light controllers (iTLCs). This information helps with optimizing road intersections and traffic flows (STONE, 2021). Newcastle University's Urban Observatory is a huge data repository collected from more than 1000 sensors for different sectors like air pollution, flooding, and even bee movements (Newcastle University, 2021). In Europe, the European Commission, through Horizon 2020, funded a program called The Smart Cities and Communities lighthouse projects. The lighthouse projects aim to gather cities, industry, and citizens to develop smart solutions for urban issues such as energy, traffic, economy, and environment. These solutions must be open source so they can be modified and reused by other cities (EU Smart Cities Information System, 2021). One of these projects is mySmartLife. It aims to support cities to have a transformation strategy for defining the city challenges and motivating smart people and smart economy into a high quality of life. mySmartLife introduces a framework for urban platform development that ensures interoperability in the platform. This framework is used to develop Masterportal platform which is used in many cities and projects. Masterportal is used in this research as a web client for the proposed urban platform.

\subsection{Urban Environment}

The urbanization lifestyle increases the difficulty of addressing environmental problems such as climate change, air pollution, and waste management (European Environment Agency, 2017). Air pollution is a severe issue as it has a direct effect on people's health. The two capital factors of air pollution are Carbon Dioxide $\left(\mathrm{CO}_{2}\right)$, and Particular Matter (PM2.5) emitted from different sectors. One considerable contributor to air pollution is the buildings sector during all stages of the building life cycle. Building construction emits $P M 2.5$ in all stages, from extracting material, moving them to the construction sites, the dumping, and the building operations. These procedures are investigated and modeled in (Muleski et al., 2005). The measure of monitoring building in all phases during building life called Life Cycle Assessment. It is a bottom-up model to estimate the condition of the building and the capability of refurbishment. This method is used in (Cuéllar-Franca and Azapagic, 2012) to estimate $\mathrm{CO}_{2}$ emissions for residential buildings during all life cycle as it was said "cuddle to crave".

Another major producer of these pollutants is traffic. It affects the quality of life in the areas around the roads in the concept of health and environment. The concentration of $P M$ with the emission rates around the roads is investigated in (Ferm and Sjöberg, 2015) using the HBEFA model to calculate PM emission factors to gain better insights about which policies and actions are needed to improve the air quality. HBEFA provides emission factors for air pollutants considering vehicle types, emissions type, year, fuel, and energy consumption (INFRAS, 2021). A similar methodology to calculate air pollutants emissions from transport is COPERT developed by European Environment Agency (EEA). The total emissions are calculated from fleet information and average speed-based emission factors (Ntziachristos and Samaras, 2021). This study uses COPERT emission factors for $\mathrm{PM} 2.5$ and $\mathrm{CO}_{2}$ to estimate the pollutants emissions from traffic information.

\section{CONCEPT OF AN INTERACTIVE ANALYTIC URBAN PLATFORM}

Nowadays, urban platforms as a concept is widely known for reflecting the state of a city into a web interface through sharing open urban data. This paper aims to expand this concept to take into account 2D/3D data integration and analysis coupled with analytic visualization and user-interacted functionalities as filtration and classification. As a case study, an urban platform is developed for the "Nordbahnhof" area in the city of Stuttgart using $3 \mathrm{D}$ city models with $2 \mathrm{D}$ traffic and environmental data. This concept depends on open standards OGC 3D container and OGC SensorThings API (STA) to deliver 3D and 2D data sets respectively from heterogeneous resources with the consideration of data interoperability, these standards are combined with web applications to process and visualize original data as well as derived information. Based on these OGC standards, this work presents two approaches to actualize the concept of an interactive analytic urban platform for the study area. First, a 2D web client is deployed using an open source framework called "Masterportal" benefiting from its pre-defined functionalities. Another approach is to develop a 3D web application to cover the needed methods to aggregate, process and visualize urban data in an interactive and analytic way. Moreover, a comparison between these two methodologies is done considering performance, processing time, efficiency, reusability, and interoperability.

An overall view of the workflow is depicted in figure 1 . The first step is to prepare 3D city models, provided in CityGML format, which must be converted into tiles for better visualization. Afterward, an OGC 3D container is developed to provide access APIs to these tilesets. Meanwhile, traffic data is collected by a scheduled job and stored in a STA server to have standard services to get traffic data. When the data and its providers are ready, two web-based platforms are implemented to visualize $3 \mathrm{D}$ city models integrated with traffic and environmental data. The final step is to evaluate the two developed platforms with the used standards according to the result, performance, timeconsuming, efficiency, and interactivity.

\subsection{Data Sets}

3.1.1 3D City Models: The 3D city models used in this project are two data sets covering "Nordbahnhof" and "Stoeckach" areas in Stuttgart city. They are formed in CityGML files include buildings, bridges, tunnels, and vegetation features. These two data sets were investigated using FME software, exporting building and bridge features then converting them into ESRI I3S and CESIUM 3D Tiles formats. 


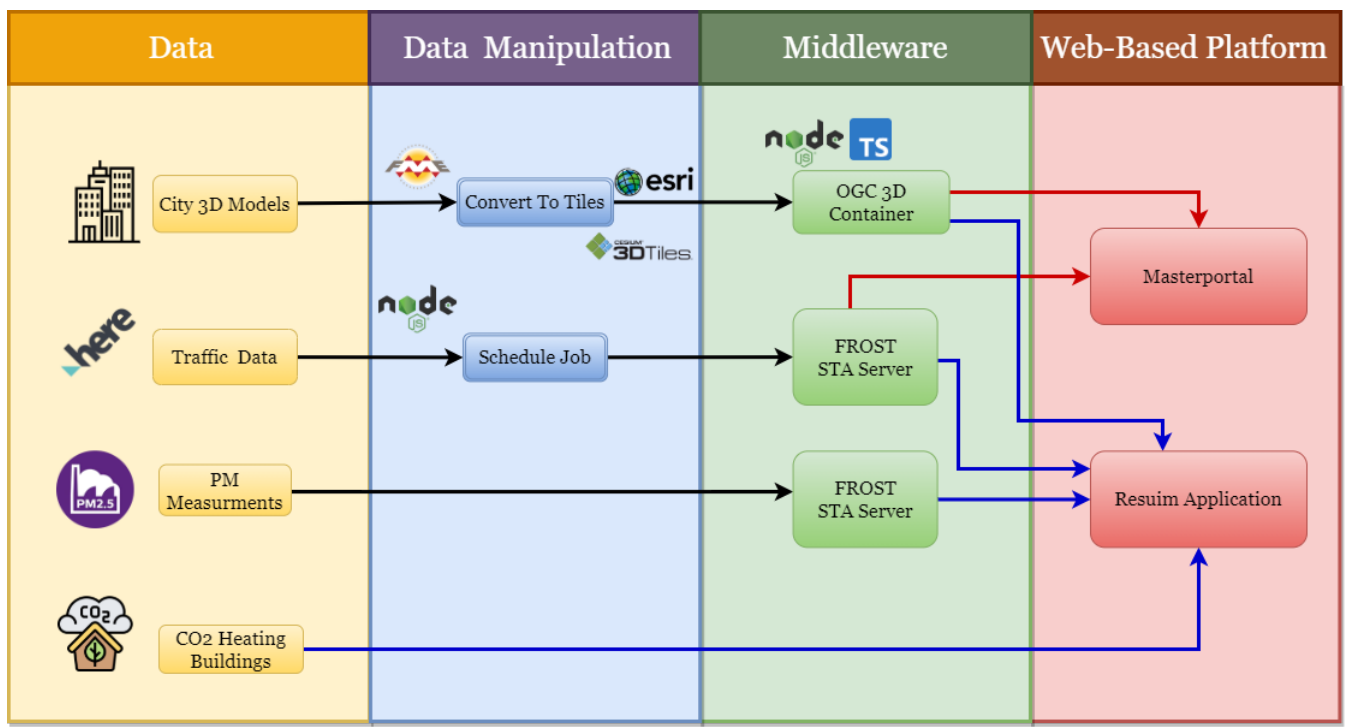

Figure 1. Overall workflow

3.1.2 Traffic Data: Traffic information for the study area is obtained through HERE traffic flow API. It gives real-time traffic data, including speed and congestion for the determined area in the API request with the geometry of the related road segments. Moreover, this traffic data is used to estimate emission rates for $\mathrm{CO}_{2}$ and $P M 2.5$ depending on average traffic speed on the road segments in the study area. The applied methodology is derived from the described method in (Cascetta et al., 2010). The formula utilizes COPERT (Ntziachristos and Samaras, 2021), which is the official method of the European Environment Agency. It depends on vehicles types shares, average speed, and segment length. Information about the vehicle types (passenger car, light drive vehicle, heavy drive vehicle, etc.), percentage of each type with the used fuel in the vehicle are required to apply this formula. Unfortunately, this information was not available when doing this work. Hence, a light version of this formula is introduced as an example of a doable manipulation of traffic data and its impact on air pollution. Some assumptions are considered to conclude the used formula (1). Firstly, All cars are passenger cars as the study area is a residential area around Stuttgart's city center and few heavy vehicle are expected to be on the roads. Secondly, the equation calculates the emission of one pollutant at a time using the pollutant emission factor gained from COPERT specification correspondent to passenger car (Ntziachristos and Samaras, 2021). Unfortunately, The exact number of cars in Stuttgart city center is not available. An estimated car count in all road segments is considered to be 62,700 vehicles per day based on a statistic mentioned on POLIS (POLIS, 2019). The formula is used to estimate $\mathrm{CO}_{2}$ and $\mathrm{PM} 2.5$ emissions on the road segments either using real-time values or average values during a period of time.

$$
P_{i, s}\left(\bar{v}_{n}^{s}\right)=L_{s} W_{s} E_{i}\left(\bar{v}_{n}^{s}\right)
$$

Where

- $P_{i}\left(\bar{v}_{n}^{s}\right)[\mathrm{g}]$ is the pollutant $i$ emission on the road sector $s$

- $L_{s}[\mathrm{~km}]$ is the length of road segment $s$

- $W_{s}$ is the number of vehicles on the road segment $s$
- $E_{i}[\mathrm{~g} / \mathrm{km}]$ is the emission factor of pollutant $i$ which is a function of the speed on road section $s$

- $\bar{v}_{n}^{s}[\mathrm{~km} / \mathrm{h}]$ is the real-time or average value of the speed on road section $s$

3.1.3 Environmental Data: The derived environmental indicators from traffic data is linked with the following environmental data:

- $\mathrm{CO}_{2}$ Simulations The residential buildings in "Stoeckach", a district from this study area, are examined using Life Cycle Assessment method to simulate $\mathrm{CO}_{2}$ emissions. The results of simulation is used to suggest refurbishment scenarios to "Stoeckach" district according to $\mathrm{CO}_{2}$ emissions form three stages construction, use and demolition (Harter et al., 2016). This project uses the simulations result for $\mathrm{CO}_{2}$ emissions from (Harter et al., 2016) which is executed using the SimStadt ${ }^{1}$ software. The result includes information about 556 residential buildings as year of construction, CityGML information, building type and $\mathrm{CO}_{2}$ emissions from space heating, domestic hot water and electrical applicants.

- PM Measurements $P M$ observations originate from a ground sensors network supported by OK Lab Stuttgart citizen science project ${ }^{2}$ measuring PM concentrations. A STA server is developed including information about the utilized air quality sensors and their locations with storing PM2.5 and $P M 10$ values in five minutes interval. In this work, 42 sensors are monitored in the study area with the observed $P M 2.5$ values. These values are used to calculate Air Quality Index (AQI) for the interested location. AQI value for $P M 2.5$ daily average can be calculated using Equation 2, where the high and low break points determined by each country (Hylton, 2016).

$$
A Q I=\frac{A Q I_{h i}-A Q I_{l o}}{\operatorname{Con}_{h i}-\operatorname{Con}_{l o}}\left(\operatorname{Con}_{i}-\operatorname{Con}_{l o}\right)+\left(A Q I_{l o}\right)
$$

Where:

\footnotetext{
1 https://simstadt.hft-stuttgart.de

2 https://luftdaten.info
} 
- Con $_{i}=$ The average value of $P M 2.5$

- $A Q I_{h i}$ and $A Q I_{l o}$ are boundaries of AQI range

- Con ${ }_{h i}$ and $C_{\text {on }}$ lo are boundaries of PM2.5 range

\begin{tabular}{|l|l|l|}
\hline & AQI & $P M 2.5 \mathrm{ug} / \mathrm{m} 3$ \\
\hline Good & $0-50$ & $0-10$ \\
\hline Moderate & $51-100$ & $11-20$ \\
\hline $\begin{array}{l}\text { Unhealthy for Sensit- } \\
\text { ive Groups }\end{array}$ & $101-150$ & $21-25$ \\
\hline Unhealthy & $151-200$ & $26-50$ \\
\hline Very Unhealthy & $201-300$ & more than 50 \\
\hline
\end{tabular}

Table 1. AQI and PM2.5 Mapping (Fraser et al., 2016)

\subsection{Data Middleware}

Towards reaching a reliable data access by different clients, using open formal standards is a key factor. On the first hand, diverse 3D data sets are provided through a server implementing OGC 3D Container standard. OGC 3D container goals to achieve smooth transition between 2D and 3D environments with the ability to get data from $2 \mathrm{D}, 2.5 \mathrm{D}$ and $3 \mathrm{D}$ resources as well as enabling 3D bounding volumes to support different formats of 3D data . 3D container concept grants a certain degree of interoperability for accessing 3D data sets from two web-based platforms in an easy and effective way (Miller et al., 2020). On the other hand, $2 \mathrm{D}$ sensor/IoT data such as traffic information and $P M$ observations are managed by using $O G C$ SensorThings API (STA) standard. It is an open, geo-spatial, and unified platform to interconnect between sensors, data and applications over the internet for more official easy way to interact and analysis the observations. STA allows developers to build different applications without concerns about multiple heterogeneous systems form different sensors. It unifies the specifications of storing observations, managing devices, and retrieving information about sensor metadata and observations result (Liang et al., 2016).

\subsection{Data Visualisation}

Two web-based applications are used for data processing and visualizing. First application is a 2D client with 3D extension which is deployed by configuring Masterportal platform. Masterportal offers a reusable base core with multiple functionalities that cities can reshaped to match their needs, it supports style design, services managing, map printing, 3D component and various other components help setting up the platform (Landesbetrieb Geoinformation und Vermessung (LGV), 2019). The second application is a 3D web client developed from scratch using RESIUM library which is built on React framework with CESIUM JS client. At the last, these two implementations are analyzed and evaluated with respect to efficiency and performance.

\section{IMPLEMENTATION}

\subsection{Traffic SensorThings API Server Implementation}

Traffic information for the study area is gained from HERE traffic flow API in JSON format then re-structure the response elements to map the entities defined in STA standard (Liang et al., 2016). In this work, we used FROST-Server an opensource implementation of SensorThings API part 1: Sensing, developed by the Fraunhofer IOSB as our SensorThings server. It is deployed using a docker container created using dockercompose tool with an YAML configuration file contains setting for the server and database (FROST Server, 2021). After setting the FROST server, an application is developed using TypeScript and Node.js. This application calls HERE traffic flow API every five minutes and wraps the response into STA entities and inserts them into the database using FROST server APIs.

\subsection{OGC 3D Container Implementation}

The implemented OGC 3D container for 3D data sets in this project has a hierarchy based on spatial extent and theme of the data. The proposed structure of 3D container's collections is consist of a main geo-volume Stuttgart with two child geo-volumes Buildings and Bridges. Each of these theme geo-volumes has child geo-volumes for the subareas "Nordbahnhof" and "Stoeckach". This hierarchy is usually defined as JSON/HTML files which keeps its implementation easy and simple. Nonetheless, it does not have any validation process for the types of the entities or their attributes. In this project, a Typescript library is developed to encapsulate the data model entities of OGC 3D container with functionality of creating these entities from JSON object with the ability of performing validation and giving the suitable exception to declare the errors in a 3D container structure. As well, a Node.js server is developed to host the proposed OGC 3D container depending on the implemented JSON files and the entities model library. The hosting server of OGC 3D container implements all geovolume API methods, the response of each method is generated using objects created from the entities model library.

\subsection{Masterportal Implementation}

Masterportal platform supports variety of data layer types with several functionalities. In this project, Masterportal is used as an urban platform for the study area in Stuttgart including the information of 3D city models and traffic data. First step to configure Masterportal is to define the map center, used projection systems, map view extent, zoom, and scale to be convenient for the data layers in configuration files config. $j$ s and config.json. Afterwards, data layers are defined with corresponding style for each layer. Masterportal has predefined types for visualizing 3D features and STA data by defining layers attributes and styles in configuration files, table 2 shows the types of data layers used is this project.

\begin{tabular}{|l|l|}
\hline Data Layer & Masterportal Layer Type \\
\hline Base map & WMS \\
\hline 3D Tiles & Tileset3D \\
\hline Traffic Observations & Sensor \\
\hline
\end{tabular}

Table 2. Layer types used in Masterportal platform

For visualizing 3D city models for the study area, four TileSet3D layers are configured with the URLs of the server hosting OGC 3D container for buildings and bridges layers. In addition, two styles are define: BuildingStyle and BridgeStyle to colorize tiles features according to their heights. Similarly, traffic data layers are styled as lines copying the road segments colorized according to the traffic speed on the roads. The roads style is updated every time a new observation is added for the road due to the implementation of MQTT protocol for "Sensor" STA layer. 


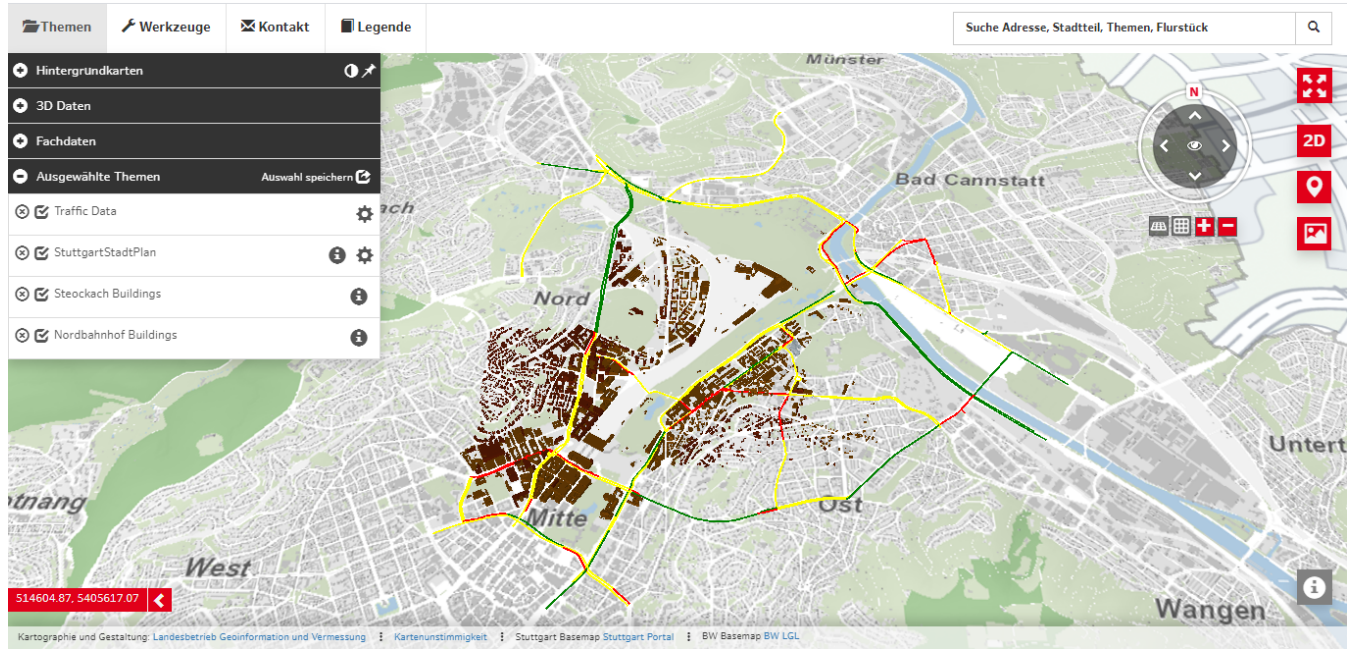

Figure 2. Masterportal interface with traffic layer and 3D city models

\subsection{D Web-Based Application Implementation}

In this application, a combination of 3D city models, traffic observations and environmental data of the study area are applied to have an analytic view of the impact of traffic in urban areas on the environment indicators like $\mathrm{CO}_{2}$ and $P M 2.5$.

4.4.1 Functionalities: The developed urban platform has the interface shown in figure 4 . It offers the following functionalities to display $3 \mathrm{D}$ and $2 \mathrm{D}$ data sets with choosing a theme to visualize. Additionally, some extra functions can be applied according to chosen theme as classification, information popup and filtering.

- 3D Container: this component accepts any valid URL for a 3D container and shows the structure of the chosen 3D container with the ability to visualize the embedded data sets in different format as CESIUM 3D Tiles or ESRI I3S

- Traffic Speed/Jam: Roads features are colorized according to traffic speed or jam. In addition, traffic themes allow the user to filter the returned data by a range of speeds, a range of jam factors, or a period of time. When data is filtered, the buildings rendered by the 3D Container are also filtered to only show the features that are within 200 meters of the visible roads because the roads as linear features has local effects on the surrounded area, as shown in figure 3 .

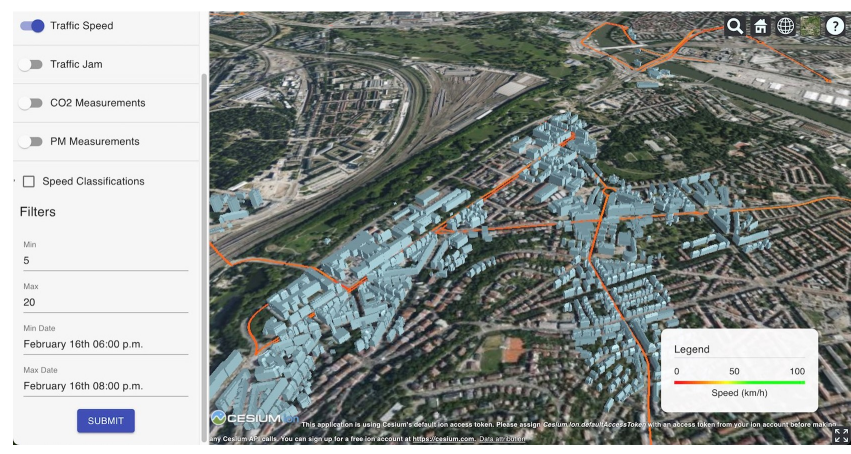

Figure 3. Filtering 2D roads with 3D buildings features

- $\mathrm{CO}_{2}$ Measurements: $\mathrm{CO}_{2}$ emissions from roads and buildings are calculated as the daily average in $[\mathrm{kg} \mathrm{CO} 2$ eq/day] and visualized with the same style, range and color to make comparison more convenient. In addition, the user can choose a specific date to show the emissions in the selected day. This limitation is a result of calculation roads $\mathrm{CO}_{2}$ emissions as daily average because we only have the average number of vehicles per day.

- PM Measurements: $P M$ emissions from roads and Luftdaten sensors are visualized. When PM measurements is chosen, a new option is activated to display the values of air quality index derived from $P M$ measurements with explanations of AQI levels with corresponding messages and warnings.

- Information Popup: Every 2D/3D displayed feature has a pop up window to show its information according to the chosen theme. As shown in figure 5, the popup for a road feature changes with changing the theme from traffic speed to $\mathrm{CO}_{2}$ measurements.

4.4.2 Components: This application has several components connected to each other. First components layer is used to query data from external resources, then it is piped down to inner layers where further processing and calculations happen. Depending on that, styling is applied to equivalent layer. Finally, the data is visualized on a CESIUM Map. The most two essential components are:

- 3D Container Component: This component was developed as a React component that would act as a 3D Container reader. The user is able to provide any valid OGC 3D Container URL, and upon submitting, the data is read with the help of the OGC 3D Container Entities Library. The collections of the 3D Container appear in a Tree List view that would recursively render the collection's children until reaching a leaf child, where its content is displayed.

- SensorThings API Adapter: The capabilities of STA server are limited to mathematical functions or filtering options but do not allow us to make complex or aggregated operations. For that reason, a component, namely "FROST API Adapter", was developed following the Adapter design pattern, which queries data from a FROST server, performs needed data aggregation, then returns the 


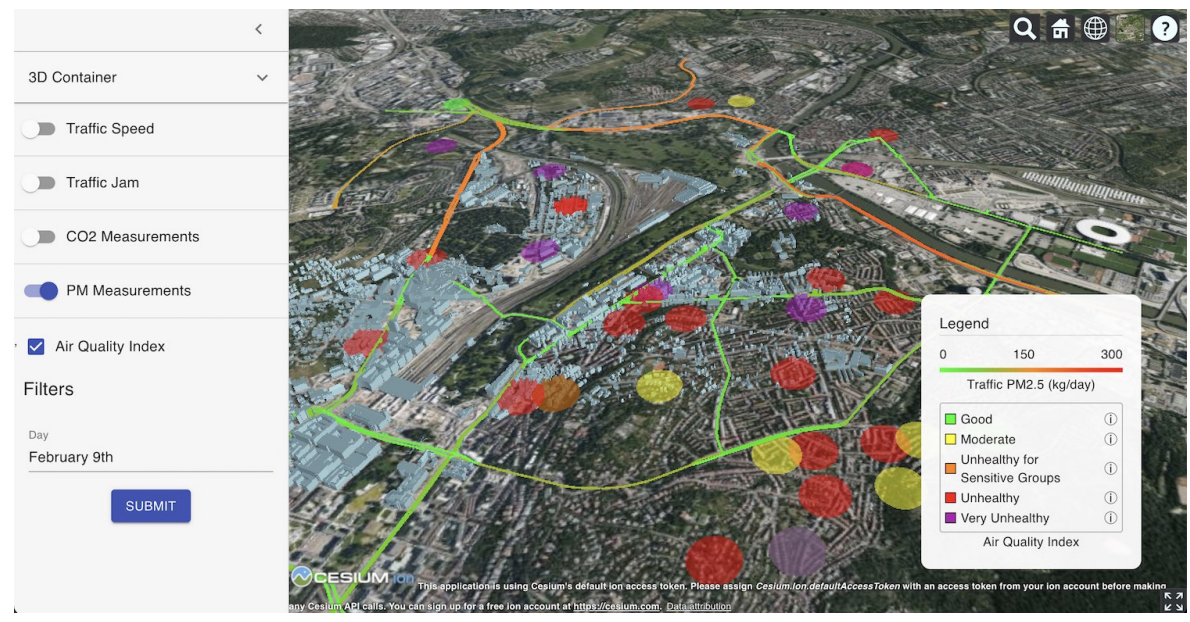

Figure 4. The proposed urban platform interface
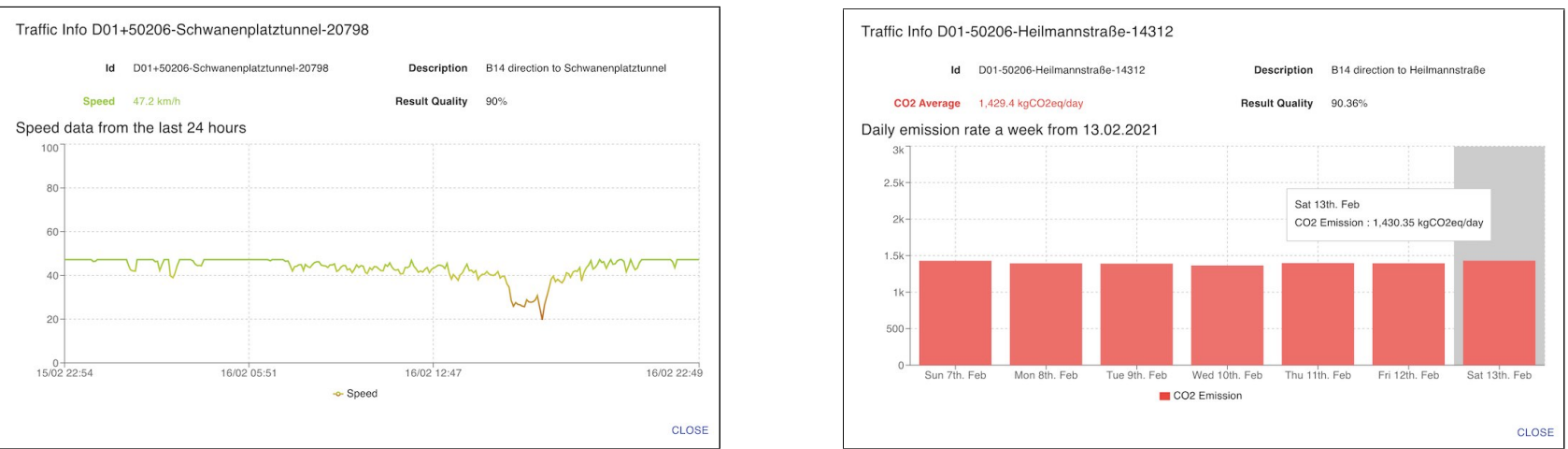

Figure 5. Information popup for a road feature according to the chosen theme traffic speed and $\mathrm{CO}_{2}$ measurements

modified data to the caller. Thus, for performing an aggregation operation, a request has to be made to retrieve all observations for all the features of interest that happened within a certain period, then perform the data aggregation within the adapter. Another limitation in a FROST server that makes this operation very slow, is that the oneto-many relationship specified between a feature of interest and an observation is only loaded lazily once needed, which means that after retrieving the features, FROST performs a query for each feature to get its observations. To overcome this issue, the adapter queries for the features only at first, then performs asynchronous calls to get the observations of each feature. This solution decreased the time needed to query and aggregate the results by about five times, since five asynchronous calls are made in parallel at a time.

\section{EVALUATION}

The proposed concept in this work is assessed on different aspects of developments: data interoperability, analysis, integration and visualization in both clients Masterportal and the developed platform.

\subsection{Data Interoperability}

The suggested concept depends on open standards to deliver 2D and 3D data sets to ensure formality when using the data. OGC 3D container standard facilitates the access to 3D data sets from both implementations with the different distributions of the content as CESIUM 3D tiles and ESRI I3S. In the meantime, 2D data sets are mapped and stored into STA standard structure. Traffic and air quality sensors generate their own measurements with their own attributes, structure, geometry and measuring units. Traffic data has the polylines of the roads with the current speed and factor jam. Meanwhile, PM2.5 data has points locations of the sensors with the measured values. STA grants formality between the heterogeneous resources and unifies the methodology of querying and processing the data despite the differences between them. The open standards utilization in this concept achieves a high degree of interoperability, making data access and manipulation done in a more simple, reliable and flexible way in both platform implementations as Masterportal and 3D web client application.

\subsection{Data Analysis}

The proposed platform applies some analyzing operations on the used data 2D and 3D to extract information about the environmental situation in the study area. All the mathematical operations, the classification and the generation of graphs for the derived values is done at run time processing. Furthermore, the proposed platform grants users the ability to perform queries of 2D and 3D data for a specific value range and/or a period of time. Data analysis and extracting added-value information can be appended to the urban platform concept to add the dimension of knowledge.

Analyzing data can be done offline and stored in a persistent database, but this could be considered as a replication of data as it can always be derived from the basic data, especially that sensing measurements has the possibility to change continuously and there is no intention to apply big data analysis on 


\begin{tabular}{|c|c|c|}
\hline & Masterportal & 3D Web based Client \\
\hline Style & Only Static & Static and Dynamic \\
\hline Styling depends on & Layer Attributes & $\begin{array}{l}\text { Layer Attributes } \\
\text { Calculated Fields } \\
\text { Relation with another layer }\end{array}$ \\
\hline 3D Buildings/Bridges Style & Height & $\begin{array}{l}\text { Different Themes: } \\
\text { - Basic color } \\
\text { - } \mathrm{CO}_{2} \text { emissions } \\
\text { - Distance from a Road }\end{array}$ \\
\hline 2D Roads Style & Speed & $\begin{array}{l}\text { Different Themes: } \\
\text { - Speed } \\
\text { - Jam Factor } \\
\text { - } \mathrm{CO}_{2} \text { Emissions } \\
\text { - } \mathrm{PM} 2.5 \text { Emissions }\end{array}$ \\
\hline
\end{tabular}

Table 3. Data styling comparison between Masterportal and developed 3D web application

them in this project. One drawback of run-time processing of STA data occurs when choosing a long time period to manipulate. The size of data becomes extremely huge and needs a long time to treat online, as STA server returns all the observations without any aggregation on the data. In this project, the traffic STA server has more than 8000 observations for each feature per month and it is possible only to fetch 1000 records per API. Thus, 156 road features require more than 2000 API requests to fetch data with a huge size of data to process.

\subsection{Data Integration}

In this concept, 2D and 3D data sets are joined using different scenarios. The $\mathrm{CO}_{2}$ emission rates from buildings in "Stoeck$a c h "$ are joined with building features as new attributes of the building. This information does not need much maintenance unless the status of the building changed due to refurbishment or destruction, which is very unlikely to happen during this project period. The second scenario for $2 \mathrm{D}$ and $3 \mathrm{D}$ data integration is through visualization, a style for data features depends on values from another data set or by a relation between different data sets. In this platform, when applying a filter on 2D road data, only buildings within 200 meters from a road are shown because roads have a local effect on the surrounded areas for the pollution emissions. The last scenario is to unify the extracted information from 2D and 3D features to make the comparison between data sets possible. Roads and buildings features have $\mathrm{CO}_{2}$ emissions rates in the same units [kg CO2 eq/year] which makes the comparison more convenient for city planners or users to get a clearer idea about emissions rates from all features. Therefore, integration between $2 \mathrm{D}$ and $3 \mathrm{D}$ data sets can be done in several methods according to the case. Merging 2D and 3D data sets on a database level is more suitable for low maintenance data. Sensing observations change frequently or have real-time values, which makes the run time integration a more efficient way.

\subsection{Data Visualization}

For data visualization, two web-based clients are used. Masterportal as a 2D client with a 3D extension and a 3D web client developed from scratch using RESIUM library. On the right hand, Masterportal is a configured platform that offers many already developed visualization functions for several types of layers, which makes it easy and simple to handle and does not need high programming skills to be configured. In addition, Masterportal has an embedded implementation for STA layers that supports both HTTP and MQTT protocols, which facilitates adding sensor observations to the platform.
On the other hand, developing a 3D web client needs some programming skills to include different layers and implement the standards into the platform. However, the developed platform from scratch gives the flexibility to develop different data processing and visualization components. 3D data sets including buildings and bridges with traffic information are visualized in Masterportal with static styles according to the embedded attributes in the features. The style is set for once by defining it in JSON format files. The mechanism of Masterportal has to be modified in order to apply interactive styles that change with user interactions or derived changeable attributes. Another downside of Masterportal is the huge memory usage when adding traffic and 3D data sets to the map at the same time, which causes the Masterportal interface to freeze. These issues of Masterportal need more investigation and development.

The 3D web-based client is developed to visualize $2 \mathrm{D}$ and $3 \mathrm{D}$ data sets in the same interface. Different dimensions data sets are queryable for the same conditions and have the same styling themes according to the same indicators. 2D and 3D data can be styled statically depending on features attributes, interactively with user queries or real-time observations, or analytically according to derived attributes or classification strategies. Furthermore, graphs for features information are added for daily average or last 24 hour charts. The 3D web-based client allows more visualization options for both 2D and 3D data sets with applying interactive and analytical styles.

\section{CONCLUSION AND FUTURE WORK}

This project shows a concept for an urban platform to perform integration between different dimensions data sets $2 \mathrm{D}$ and $3 \mathrm{D}$ with applying analytic operations on the data to gain added value information about the city with data visualization in two platforms. Firstly, Data manipulation is a significant element in the urban platform involving integration and analyzing to extract knowledge, helping with understanding the city-state and how to better the quality of life in the city. For example, the environmental emissions rates included in the developed platform could be considered as indicators that lead the urban planning to focus on specific areas in the city or to concentrates on specific issues. Likewise, Adding buildings 3D models to urban platforms provide a more precise idea about the city situation. Additionally, a combination between 2D and 3D data sets complete the analytic view of the different domains in urban areas.

Secondly, a comparison between two used clients: Masterportal and a developed 3D web application has been made. 
Masterportal is a simple platform that can be used easily to display different types of data $2 \mathrm{D}$ and $3 \mathrm{D}$, but it lacks the ability to analyze the displayed data and has a limited degree of interactive options with the end-user. Moreover, it does not support interaction between the layers; these functionalities can be added to Masterportal as it is an open-source platform. The developed $3 \mathrm{D}$ client is a flexible and extendable platform. It is developed in an abstract and generic way to accept data from different resources to analyze and visualize data sets with the capability to have a mutual visualization between $2 \mathrm{D}$ and $3 \mathrm{D}$ data sets. In addition, the platform provides user-interacted functions but these functionalities need to be evaluated depending on user users experiences and feedbacks to establish the long-term improvement of the platform.

Finally, it must be considered that there are some limitations to this project. Data availability is a major concern; the calculations of pollutants emissions would be more precise when using detailed information about traffic like the types of vehicles and road specifications. Moreover, PM2.5 propagation between roads and buildings areas can be calculated using the Land Use Regression model with spatial interpolation to predict $P M 2.5$ concentration in the area depending on several parameters: distance from roads, buildings height, vegetation ratio and land use (Ghassoun et al., 2015).

\section{REFERENCES}

Brutti, A., de sabbata, P., Frascella, A., Gessa, N., Ianniello, R., Novelli, C., Pizzuti, S., Ponti, G., 2019. Smart City Platform Specification: A Modular Approach to Achieve Interoperability in Smart Cities: Technology, Communications and Computing. $25-50$.

Cascetta, E., Punzo, V., Bonne, P., 2010. Impact on vehicle speeds and pollutant emissions of a fully automated section speed control scheme on the Naples urban motorway. 19th Intelligent Transport Systems World Congress, ITS 2012.

Chamoso, P., De La Prieta, F., 2015. Swarm-Based Smart City Platform: A Traffic Application. Advances in Distributed Computing and Artificial Intelligence Journal, 4, 89-98.

Cuéllar-Franca, R., Azapagic, A., 2012. Environmental impacts of the UK residential sector: Life cycle assessment of houses. Building and Environment, 54, 86-99.

EU Smart Cities Information System, 2021. Smart Cities and Communities Lighthouse projects. https://smartcitiesinfosystem.eu/scc-lighthouse-projects.

European Environment Agency, 2017. Urban Environment. https://www.eea.europa.eu/themes/sustainabilitytransitions/urban-environment.

Ferm, M., Sjöberg, K., 2015. Concentrations and emission factors for PM2.5 and PM10 from road traffic in Sweden. Atmospheric Environment, 119, 211-219.

Fraser, A., Loader, A., Pang, Y., Stewart, R., Xiao, X., Whitehead, C., 2016. Services to develop an EU Air Quality Index.

FROST Server, 2021. FROST Server. https://fraunhoferiosb.github.io/FROST-Server/.
Ghassoun, Y., Ruths, M., Löwner, M.-O., Weber, S., 2015. Intra-urban variation of ultrafine particles as evaluated by process related land use and pollutant driven regression modelling. Science of The Total Environment, 536, 150-160.

Harter, H., Weiler, V., Eicker, U., 2016. Developing a roadmap for the modernisation of city quarters - Comparing the primary energy demand and greenhouse gas emissions. Building and Environment, 112.

Hernandez, J., Garcia, R., Schonowski, J., Atlan, D., Chanson, G., Ruohomäki, T., 2020. Interoperable Open Specifications Framework for the Implementation of Standardized Urban Platforms. Sensors, 20, 2402.

Hylton, M., 2016. AQI Calculations Overview- Ozone, PM2.5 and PM10. https://forum.airnowtech.org/t/aqi-calculationsoverview-ozone-pm2-5-and-pm10/168.

INFRAS, 2021. The Handbook of Emission Factors for Road Transport (HBEFA). https://www.hbefa.net/e/index.html.

Landesbetrieb Geoinformation und Vermessung (LGV), 2019. Masterportal in Hamburg. https://www.masterportal.org.

Lea, R., Blackstock, M., 2015. City Hub: A Cloud-Based IoT Platform for Smart Cities. Proceedings of the International Conference on Cloud Computing Technology and Science, CloudCom, 2015, 799-804.

Liang, S., Huang, C.-Y., Khalafbeigi, T., 2016. OGC SensorThings API Documentation. http://docs.opengeospatial.org/is/15-078r6/15-078r6.html.

Miller, T., Trenum, G., Lieberman, J., 2020. 3D Data Container Engineering Report. https://docs.ogc.org/per/20-029.html.

Miu, M., Zhang, X., Dewan, M. A. A., Wang, J., 2018. Development of Framework for Aggregation and Visualization of Three-Dimensional (3D) Spatial Data. Big Data and Cognitive Computing, 2(2). https://www.mdpi.com/2504-2289/2/2/9.

Muleski, G. E., Jr., C. C., Kinsey, J. S., 2005. Particulate Emissions from Construction Activities. Journal of the Air \& Waste Management Association, 55(6), 772-783.

Newcastle University, 2021. UK Urban Observatory. https://www.ncl.ac.uk/who-we-are/vision/urban-observatory/.

Ntziachristos, L., Samaras, Z., 2021. EMEP/EEA air pollutant emission inventory guidebook. https://www.eea.europa.eu/ds_resolveuid/SHNJDK8413.

POLIS, 2019. Baden-Württemberg: The LEZ of Stuttgart delivers good results. https://www.polisnetwork.eu/news/badenwurttemberg-the-lez-of-stuttgart-delivers-good-results/.

STONE, T., 2021. Urban Data Access Platform (UDAP) now fully operational for complete control of intersections across the Netherlands. https://www.traffictechnologytoday.com/news/its/launchof-dutch-urban-data-access-platform-set-to-optimise-trafficflow.html.

Tobias Brandt, M. v. O., Sheombar, H., 2020. Digitally managed cities of the future - how close are we? https://discovery.rsm.nl/articles/436-digitally-managedcities-of-the-future-how-close-are-we/.

URBANET, 2016. The world urban population-Infographics. https://www.urbanet.info/world-urban-population/. 